\title{
Studies on birds of Biratnagar Sub-Metropolis, Nepal
}

\author{
Kanchan Jha and Bharat Raj Subba \\ Department of Zoology, Post Graduate Campus, T.U., Biratnagar, Nepal \\ E-mail: subbabharat@yahoo.com
}

\begin{abstract}
Biratnagar sub-metropolis is a good habitat for several bird species but in the past three decades drastic changes have taken place in the environment of this place because of anthropogenic activities. Several commonly visible birds' species do not turn out at present. Habitats of birds have shrunk here in such a way that very limited species of birds have become successful to show representatives in this situation. Considering gradual loss of habitats and their inhabitants, an attempt was made to study the present bird status. In the observation of seven months, seventy two species of birds belonging to thirty two families were recorded. The birds recorded, were residents $(72.17 \%)$, winter visitors $(23.59 \%)$ and summer visitors $(4.16 \%)$.
\end{abstract}

Key words: Birds, Biratnagar, status.

\section{Introduction}

Biratnagar $\left(26^{\circ} 26^{\prime} \mathrm{N}\right.$ to $\left.087^{\circ} 16^{\prime} \mathrm{E}\right)$ is the second largest city situated in the eastern region of Nepal. Sub-tropical type of climate prevails here. The vegetation is of mixed type and planted trees are in scattered state. There are wetlands, rivers, man-made ponds for the habitats of both aquatic and terrestrial birds. Birds are more glorious animal of recreational value than others. They have always been sources of recreation for human beings. Their attractive beautiful plumage, melodious songs and artistic behaviour never fail to make the nature fascinating. Birds have been close friends of human beings in varied ways. They play many important roles to keep the ecosystem balanced. 865 species of birds have been recorded in Nepal.

The present study is focused on preparation of a checklist of birds and their present status. The work also encompasses the study of anthropogenic impacts on birds habitats and their feeding grounds. The present report is the outcome of seven months (Oct, 2011-March, 2011) regular observations. Several ornithologists have contributed to the bird of Nepal, among them, Ali and Ripley (1968-74), Fleming et al. (1984), Subba (1994), Grimmett et al. (2000), Shrestha (2001), Baral and Inskipp (2001) and Baral (2005) are cited here.

\section{Study site}

Five main sites Tintolia- Balwahi $\left(26^{\circ} 26^{\prime} 32.4^{\prime \prime} \mathrm{N}, 087^{\circ} 18^{\prime} 09.4^{\prime \prime}\right.$ E), Baijnathpur, Ginaghat $\left(26^{\circ} 29^{\prime} 59.8^{\prime \prime} \mathrm{N}, 087^{\circ} 17^{\prime} 53.2^{\prime \prime} \mathrm{E}\right)$, Keshaliyakhola $\left(26^{\circ} 27^{\prime} 00^{\prime \prime} \mathrm{N}, 087^{\circ} 14.4^{\prime}\right.$ $\left.48.5^{\prime \prime} \mathrm{E}\right)$, Dudhfarm $\left(26^{\circ} 29^{\prime} 57.6^{\prime \prime} \mathrm{N}, 087^{\circ} 16^{\prime} 44.5^{\prime \prime} \mathrm{E}\right)$ and Old airport $\left(26^{\circ} 25^{\prime} 40.8^{\prime \prime} \mathrm{N}\right.$, $087^{\circ} 16^{\prime} 34.3^{\prime \prime} \mathrm{E}$ ) were selected for the study. These are the main places in Biratnagar to watch birds. 


\section{Methodology}

Birds were observed starting from 6.00 a.m. to 8.00 a.m. using binocular $(8 \times 42)$, Canon digital camera and GPS (etrex). Direct counting method was applied. Identification of the birds was done with the help of available books and literature (Flemming et al., 1984; Ali \& Rapley, 1987; Grimett et al., 2000).

\section{Results and Discussion}

Altogether seventy two species of birds belonging to 32 families were recorded (Table 1). Among them Muscicapidae had the highest number of species representatives (eight) followed by Corvidae and Passeridae with five representatives. Similarly Columbridae, Ardeidae, Cuculidae, Scolopacidae and Sylviidae lied in the third position, each family represented by four species. Sturnidae had three representatives. Hirundinide, Phalacrocoracidae, Pycnonotidae, Laniidae, Psittacidae and Ciconidae were represented by two species. Rhalidae, Centropodidae, Alcedinidae Megalaimidae, Picidae, Accipitridae, Alaudidae, Oriolidae, Apopidae, Charadriidae, Coraciidae, Upupidae, Nectariniidae, Threskiornithidae, and Anatidae had least number of representatives. Among seventy two species of birds recorded, 72.17\% were resident, $23.59 \%$ winter visitor and $4.16 \%$ summer visitor. Similarly on the basis of population, $56.90 \%$ were common, $31.92 \%$ fairly common and $11.10 \%$ uncommon (Figs. 1-2).

Up to three decades back, in Biratnagar, bird habitats were almost safe. Here, one could observe more than fifty species of birds in two hours bird watching in any season. Subba (1994) recorded 93 species of birds in the same locality and time but this year only 72 species were recorded on regular observations. Many species like, Leptoptilos dubius, Ciconia episcopus, Gypus indicus, Gyps fulvus, Milvus migrans, Circus melanoleucos, Charadrius dubius, Tringa nebularia, Burhinus oedicnemus, Capella gallinago, Athene brama, Apus affinis. Alcedo meninting, Pelargopsis capensis, Alcedo atthis, Emberiza aureola, Ploceus philippinus, Megalaima haemacephala, Dendrocopus nanus did not turn out this time. This is due to either habitat shrinkage or anthropogenic impacts or may be because of both reasons. Within one decade urbanization has touched the peak here. Marshy lands have been encroached almost completely. River waters have become polluted and are not suitable for wetland birds. People are really apathy for the conservation of bird habitat and their nesting and feeding sites. The population of each and every species of bird has been declining markly. If birds safeguard activities will not be made popular in time and made people realize why birds have to be protected there lies every possibility of declining birds population as well as species. If conservation of birds will remain restricted to slogan only then time may come once when it will be too late for safeguard of birds.

Table 1. Checklist of Birds.

\begin{tabular}{cllcc}
\hline SN Family & Scientific name & Common name & \multicolumn{2}{c}{ Status Remark } \\
\hline 1 Rhalidae & Amaurornis phoenicurus & White breasted Waterhen & $\mathrm{R}$ & $\mathrm{C}$ \\
2 Corvidae & Corvus splendens & House Crow & $\mathrm{R}$ & FC \\
& Dendrocitta vagabunda & Indian Treepie & $\mathrm{R}$ & $\mathrm{C}$ \\
& Corvus macrohynchos & Jungle Crow & $\mathrm{R}$ & FC \\
& Dicrurus macrocerus & Black Drongo & $\mathrm{R}$ & FC \\
& Aegithina tiphia & Common Iora & $\mathrm{R}$ & $\mathrm{C}$ \\
& Streptopelia chinensis & Spotted Dove & $\mathrm{R}$ & FC
\end{tabular}




\begin{tabular}{|c|c|c|c|c|}
\hline & Columba livia & Pigeon & $\mathrm{R}$ & FC \\
\hline & Streptopelia decaoto & Indian Ring Dove & $\mathrm{R}$ & FC \\
\hline & Streptopelia tranquebarica & Red-collared Dove & $\mathrm{R}$ & $\mathrm{C}$ \\
\hline \multirow[t]{2}{*}{4 Pycnonototidae } & Pycnonotus cafer & Redvented Bulbul & $\mathrm{R}$ & FC \\
\hline & Pycnonotus jacosus & Red whiskered Bulbul & $\mathrm{R}$ & FC \\
\hline \multirow[t]{2}{*}{5 Laniidae } & Lanius cristatus & Brown Shrike & WV & $\mathrm{C}$ \\
\hline & Lanius schach & Long tailed Shrike & WV & $\mathrm{C}$ \\
\hline \multirow[t]{3}{*}{6 Sturnidae } & Acridotheres tristis & Common Myna & $\mathrm{R}$ & FC \\
\hline & Sturnus contra & Pied Myna & $\mathrm{R}$ & FC \\
\hline & Acridotheres ginginianus & Bank Myna & $\mathrm{R}$ & FC \\
\hline \multirow[t]{4}{*}{7 Ardeidae } & Bubulcus ibis & Cattle Egret & $\mathrm{R}$ & FC \\
\hline & Ardeola grayii & Indian pond Heron & $\mathrm{R}$ & FC \\
\hline & Mesophoyx intermedia & Intemediate Egret & $\mathrm{R}$ & $\mathrm{C}$ \\
\hline & Nycticorax nycticorax & Black-crowned Nightheron & $\mathrm{R}$ & $\mathrm{C}$ \\
\hline \multirow[t]{8}{*}{8 Muscicapidae } & Rhipidura albicollis & $\begin{array}{l}\text { White throated Faintail } \\
\text { Flycatcher }\end{array}$ & $\mathrm{R}$ & $\mathrm{C}$ \\
\hline & Culicicapa ceylonesis & Grey headed Flycatcher & WV & $\mathrm{C}$ \\
\hline & Ficedula parva & Red throated Flycatcher & WV & $\mathrm{C}$ \\
\hline & Phoenicurus ochuros & Black red Start & $\mathrm{R}$ & $\mathrm{C}$ \\
\hline & Luscinia pectoralis & White tailed Rubythroat & $\mathrm{R}$ & $\mathrm{C}$ \\
\hline & Saxicola torquata & Collard Bushchat & WM & $\mathrm{C}$ \\
\hline & Turdus ruficollis atrogularis & Black thrush & WV & UC \\
\hline & Copsychus saularis & Oriental Magpie Robin & $\mathrm{R}$ & FC \\
\hline \multirow[t]{4}{*}{9 Cuculidae } & Eudynamys scolopacea & Asian koel & $\mathrm{R}$ & FC \\
\hline & Clamator jacobines & Pied crested cockoo & SV & $\mathrm{C}$ \\
\hline & Cuculus canorus & Eurasian Cuckoo & SV & $\mathrm{C}$ \\
\hline & Cuculus micropterus & Indian Cuckoo & SV & $\mathrm{C}$ \\
\hline 10 Centropodidae & Centropus sinensus & Great Caucal & $\mathrm{R}$ & FC \\
\hline \multirow[t]{2}{*}{11 Alcedinidae } & Halcyon smyrnensis & White breated Kingfisher & $\mathrm{R}$ & $\mathrm{C}$ \\
\hline & Ceryle rudis & Lesser pied Kingfisher & $\mathrm{R}$ & $\mathrm{UC}$ \\
\hline 12 Megalaimidae & Megalaima asiatica & Blue throated Barbet & $\mathrm{R}$ & $\mathrm{C}$ \\
\hline 13 Picidae & Dinopium benghalense & $\begin{array}{l}\text { Lesser golden backed } \\
\text { Woodpecker }\end{array}$ & $\mathrm{R}$ & $\mathrm{C}$ \\
\hline 14 Accipitridae & Accipiter niscus & Eurasian sparrow hawk & WV & $\mathrm{C}$ \\
\hline 15 Alaudidae & Mirafra assamica & Lark & WV & $\mathrm{C}$ \\
\hline 16 Oriolidae & Oriolus xanthornus & Black headed Oriole & $\mathrm{R}$ & $\mathrm{C}$ \\
\hline \multirow[t]{5}{*}{17 Passeridae } & Anthus rufulus & Paddyfield Pipit & $\mathrm{R}$ & $\mathrm{C}$ \\
\hline & Motacilla alba ducunensis & $\begin{array}{l}\text { Grey headed Wagtail } \\
\text { (blackbacked form) }\end{array}$ & $\mathrm{R}$ & $\mathrm{C}$ \\
\hline & Motacilla cinerea & $\begin{array}{l}\text { Pied Wagtail (Grey } \\
\text { backed form) }\end{array}$ & $\mathrm{R}$ & $\mathrm{C}$ \\
\hline & Passer domesticus & House Sparrow & $\mathrm{R}$ & FC \\
\hline & Motacilla muderaspatensis & White browed Wagtail & $\mathrm{R}$ & $\mathrm{C}$ \\
\hline \multirow[t]{2}{*}{18 Phalacrocoracidae } & ePhalacrocorax niger & Little Cormorant & $\mathrm{R}$ & $\mathrm{UC}$ \\
\hline & Phalacrocorax carbo & Great Cormorant & $\mathrm{R}$ & $\mathrm{UC}$ \\
\hline 19 Apodidae & Cypriurus balasiensis & Palm swift & $\mathrm{R}$ & $\mathrm{C}$ \\
\hline 20 Charadriidae & Vanellus indicus & Redwattled Lapwing & $\mathrm{R}$ & $\mathrm{C}$ \\
\hline \multirow[t]{2}{*}{21 Meropidae } & Merops orientalis & Green Bee eater & WV & $\mathrm{C}$ \\
\hline & Merops philippinus & Bluetailed Bee eater & WV & $\mathrm{C}$ \\
\hline \multirow[t]{2}{*}{22 Hirundinidae } & Riparia riparia & Sand Martin & $\mathrm{R}$ & $\mathrm{C}$ \\
\hline & Delichon nipalensis & Nepal Martin & $\mathrm{R}$ & $\mathrm{C}$ \\
\hline \multirow[t]{4}{*}{23 Scolopacidae } & Calidris minuta & Little Stint & WV & $\mathrm{C}$ \\
\hline & Calidris temminckii & Temminck's Stint & WV & $\mathrm{C}$ \\
\hline & Tringa terek & Terreck Sandpiper & WV & $\mathrm{C}$ \\
\hline & Actitis hypoleucos & Common Sandpiper & WV & $\mathrm{C}$ \\
\hline
\end{tabular}




\begin{tabular}{lllcc} 
24 Coraciidae & Coracias benghalensis & Indian Roller & $\mathrm{R}$ & $\mathrm{UC}$ \\
25 Upupidae & Upupa epops & Hoopoe & $\mathrm{R}$ & $\mathrm{C}$ \\
26 Psittacidae & Psittacula himalayana & Slatyheaded Parakeet & $\mathrm{R}$ & $\mathrm{UC}$ \\
& Psittacula krameri & Rose ring Parakeet & $\mathrm{R}$ & $\mathrm{UC}$ \\
27 Nectariniidae & Nectarinia asiatica & Purple Sunbird & $\mathrm{R}$ & $\mathrm{FC}$ \\
28 Sylviidae & Phylloscopus fuscatus & Dusky Warbler & $\mathrm{WV}$ & $\mathrm{C}$ \\
& Turdoides striatus & Jungle Babbler & $\mathrm{R}$ & FC \\
& Orthotomus suctorius & Tailor Bird & $\mathrm{R}$ & FC \\
& Phylloscopus trochiloides & Greenish warbler & $\mathrm{WV}$ & $\mathrm{C}$ \\
29 Therskiornithidae & Pseudibis papillosa & Black Ibis & $\mathrm{R}$ & $\mathrm{C}$ \\
30 Ciconiidae & Anastomus oscitans & Asian Openbill & $\mathrm{R}$ & FC \\
& Leptoptilos javanicus & Lesser Adjutant & $\mathrm{R}$ & FC \\
31 Anatidae & Dendrocygna javanica & Lesser whistling teal & $\mathrm{R}$ & FC \\
32 Accipitridae & Haliaeetus leucoryphus & Pallas's fishing eagle & WV & UC \\
\hline
\end{tabular}

$\mathrm{R}=$ Residental, $\mathrm{WV}=$ Winter visitor, $\mathrm{FC}=$ Fairly common, $\mathrm{LC}=$ Local resident, $\mathrm{SV}=$ Summer visitor, $\mathrm{UC}=$ Uncommon

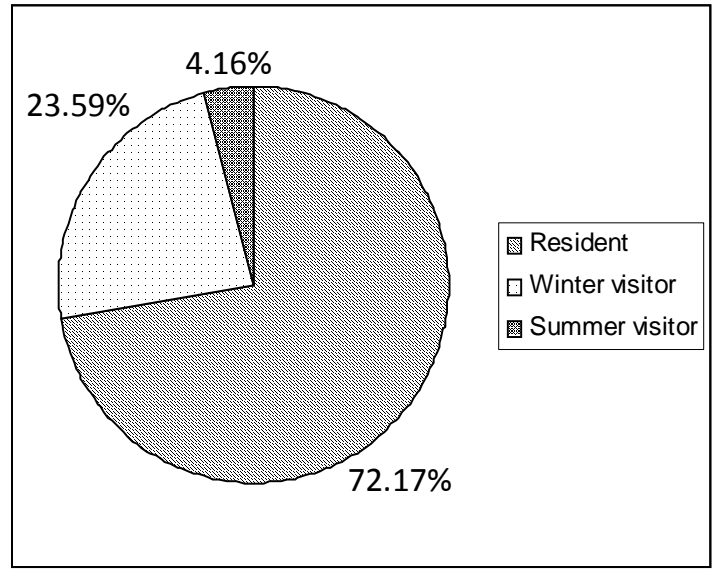

Figure 1. Behavior of birds.

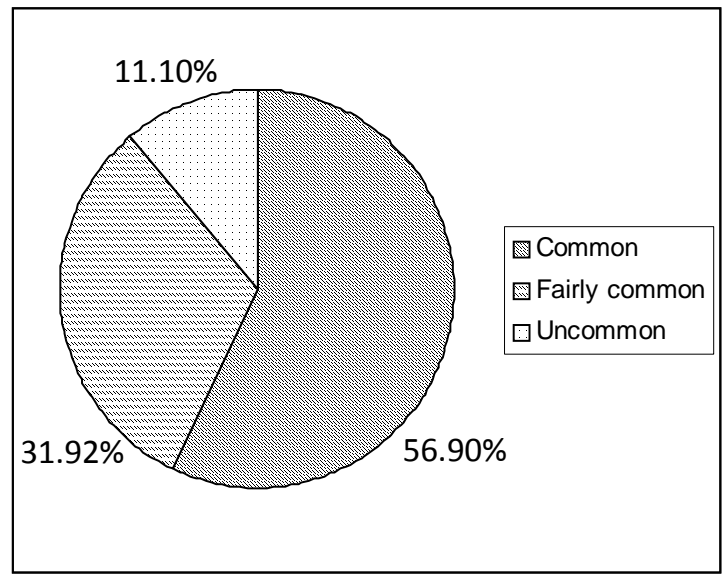

Figure 2. Population status of birds

\section{References}

Ali, S. \& S.D. Riply. 1987. Compact handbook of birds of India and Pakistan together with those of Bangladesh, Nepal, Bhutan and Srilanka. Oxford University press, Delhi.

Baral, H.S. \& C. Inskipp. 2001. Important bird areas in Nepal: a report to the Royal society for the Protection of Birds, U.K. Kathmandu: Bird Conservation Nepal.

Baral, H.S. 2005. Survey for Lesser Adjutant Leptoptilos javanicus in and around Koshi Tappu wildlife Reserve, Nepal. Forktail 21: 190-193.

Flemming, Sr. R.L., R.L. Flemming Jr. \& L.S. Bandel. 1984. Birds of Nepal. Nature Himalayas, Kathmandu, Nepal.

Grimmett, R., C. Inskipp \& T. Inskipp. 2000. Birds of Nepal. Christopher Helm A\&C Black, London.

Shrestha, T.K. 2001. Birds of Nepal. Kathmandu, Nepal.

Subba, B.R. 1994. Checklist of Birds of Biratnagar, News letter for Birdwatchers. Published biomonthly by Sridhar at Navbharath Enterprises, Sesadripuram, Bangalore 560 020, India 34(6): 128-129. 